\title{
On Model Matching Problems of Input-Output Switching Systems
}

\author{
Mohammad Naghnaeian and Petros G. Voulgaris
}

\begin{abstract}
In this paper we consider the model matching problem where linear switched systems are involved. We focus on the worst-case $l_{\infty}$ induced norm and consider the problem when the involved systems have independent or matched switching sequences. We show that a switched compensation which can switch independently of the plant has no advantage over an LTI compensation. Moreover, if the plant is strictly causal, even a switched compensation which has the same switching as the plant does not provide a better performance over an LTI compensation. We further generalize the results of [1] to MIMO systems and present an exact expression to compute the worst-case $l_{\infty}$ induced norm. Using that, one can cast a class of model matching problems as a Linear Programming.
\end{abstract}

\section{INTRODUCTION}

Linear switched systems have been studied in a great deal of literature. They arise in many contexts. One can refer to [2], [3], [4], and [5] to see examples of the works done in this field. Recently, in [6], a typical model matching problem was considered with the involving systems being output switching and the underlying norm being $l_{\infty}$-induced or $\mathscr{H}_{2}$. The authors studied a problem of the form

$$
\inf _{Q} \sup _{\left(\sigma_{H}, \sigma_{U}, \sigma_{Q}\right)}\|H-U Q\|,
$$

where $H, U$, and $Q$ are output switching systems associated respectively with switching sequences $\sigma_{H}, \sigma_{U}$, and $\sigma_{Q}$. It was shown that in the case of independent switching or partially matched switching, i.e. $\sigma_{H}=\sigma_{U} \neq$ $\sigma_{Q}$, an output switching $Q$ cannot out-perform an LTI $Q$.

In this paper, we consider a similar problem and extend the results of [6] to show that a switched linear compensation of any type (not only output switching) cannot lead to a better performance over an LTI compensation if

1) the compensator can switch independently of the plant, or

2) the plant is strictly causal.

M. Naghnaeian is a PhD candidate with the Mechanical Science and Engineering Department, University of Illinois, Urbana, IL, USA naghnae2@illinois.edu

P. G. Voulgaris is with the Aerospace Engineering Department and the Coordinated Science Laboratory, University of Illinois, Urbana, IL, USA voulgarieillinois.edu

This work was supported in part by the National Science Foundation under NSF Award NSF ECCS 10-27437 and AFOSR under award AF FA 9550-12-1-0193
Furthermore, we consider the case of matched switching with $H$ and $U$ being output switching and $Q$ being input switching. Then, the problem under study can be cast as linear programming using an exact expression derived for the $l_{\infty}$ gain of the input-output switching system.

This paper is organized as follows. In Section II, the notations, preliminaries, and the problem formulation are given. In Sections III and IV, we present our main results for the case of independent and matched switching sequences. We also show that to solve some class of the problems we need to compute the worst-case $l_{\infty}$ induced norm of the input-output switched systems which is done in Section V. Finally, we conclude in the last section.

\section{Notations, Preliminaries, AND Problem SETUP}

In this paper, $\mathbb{Z}_{+}$denotes the set of the non-negative integers. Let $T, M$, and $(g(k))_{k=0}^{\infty}$ be an operator, a matrix, and a sequence, respectively. Then by $\|T\|$, $|M|_{1},\|g\|_{1}$, and $\|g\|_{\infty}$ we mean the $l_{\infty}$ induced norm of $T, l_{1}$ norm of the matrix $M, l_{1}$, and $l_{\infty}$ norm of the sequence $g$, respectively, whenever they are defined. $\Lambda^{k}$ denotes the delay operator for $k \in \mathbb{Z}_{+}$; for any sequence $g=\{g(0), g(1), \ldots\}$,

$$
\Lambda^{k} g=\{\underbrace{0, \ldots, 0}_{k \text { zeros }}, g(0), g(1), \ldots\},
$$

and

$$
\Lambda^{-k} g=\{g(k), g(k+1), \ldots\} .
$$

Let $\mathscr{L}_{T I}$ denote the set of $l_{\infty}$-bounded Linear Time Invariant (LTI) systems. Furthermore, let $\mathscr{S}$ denotes a class of $l_{\infty}$-bounded linear switched systems, where each element $H_{\sigma}$ of $\mathscr{S}$ is associated with a switching sequence $\sigma: \mathbb{Z}_{+} \rightarrow \mathbb{Z}_{N}:=\{1,2, \ldots, N\}$ and a sequence of 4-tuples $\left(A_{\sigma(k)}, B_{\sigma(k)}, C_{\sigma(k)}, D_{\sigma(k)}\right)_{k=0}^{\infty}$ with elements in the set

$$
\left\{\left(A_{i}, B_{i}, C_{i}, D_{i}\right): i \in \mathbb{Z}_{N}\right\} .
$$

Moreover, $H_{\sigma}$ admits the realization

$$
H_{\sigma}:\left\{\begin{array}{c}
x(t+1)=A_{\sigma(t)} x(t)+B_{\sigma(t)} u(t) \\
y(t)=C_{\sigma(t)} x(t)+D_{\sigma(t)} u(t)
\end{array},\right.
$$

where $u \in \mathbb{R}^{m}, x \in \mathbb{R}^{n}$, and $y \in \mathbb{R}^{p}$ are input, state, and output of the system, respectively, and $A_{i}, B_{i}, C_{i}$, and $D_{i}$ are matrices with appropriate dimensions for all $i \in \mathbb{Z}_{N}$. 
We denote by $\mathscr{S}_{I O}$ a subset of $\mathscr{S}$ that contains only input-output switching systems, that is every $G_{\sigma} \in \mathscr{S}_{I O}$ admits the realization

$$
G_{\sigma}:\left\{\begin{array}{l}
x(t+1)=A x(t)+B_{\sigma(t)} u(t) \\
y(t)=C_{\sigma(t)} x(t)+D_{\sigma(t)} u(t)
\end{array},\right.
$$

where $A:=A_{1}=A_{2}=\ldots=A_{N}$ is a constant Schur matrix. Let $\mathscr{S}_{O}$ and $\mathscr{S}_{I}$ denote subsets of $\mathscr{S}_{I O}$ that contain only output, and input switching systems, respectively. That is for every $P_{\sigma} \in \mathscr{S}_{O}$ and $Q_{\sigma} \in \mathscr{S}_{I}$, we have

$$
\begin{aligned}
P_{\sigma} & =\left(\begin{array}{c|c}
A & B \\
\hline C_{\sigma(t)} & D_{\sigma(t)}
\end{array}\right), \text { with } A \text { and } B \text { constant, } \\
Q_{\sigma} & =\left(\begin{array}{c|c}
A & B_{\sigma(t)} \\
\hline C & D_{\sigma(t)}
\end{array}\right), \text { with } A \text { and } C \text { constant. }
\end{aligned}
$$

Define the switching operator $S_{\sigma}$ as

$$
S_{\sigma}(t)=[0,0, . ., 0, I, 0, . ., 0],
$$

where identity is at $\sigma(t)^{t h}$ entry. The dimension of $S_{\sigma}(t)$ will be clear from its context. Using this operator any $G_{\sigma} \in \mathscr{S}_{I O}$ can be written as

$$
G_{\sigma}=S_{\sigma}\left[\begin{array}{cccc}
G_{11} & G_{12} & \cdots & G_{1 N} \\
G_{21} & G_{22} & & \\
\vdots & & \ddots & \\
G_{N 1} & G_{N 2} & \cdots & G_{N N}
\end{array}\right] S_{\sigma}^{*},
$$

where

$$
G_{i j}=\left(\begin{array}{c|c}
A & B_{j} \\
\hline C_{i} & D_{i}
\end{array}\right) \in \mathscr{L}_{T I}, \text { for } i \in \mathbb{Z}_{N},
$$

and

$$
S_{\sigma}^{*}(t)=S_{\sigma}(t)^{T}, \text { for } t \in \mathbb{Z}_{+} .
$$

Therefore, for any $P_{\sigma} \in \mathscr{S}_{O}$ and $Q_{\sigma} \in \mathscr{S}_{I}$, we have

$$
\begin{gathered}
P_{\sigma}=S_{\sigma}\left[\begin{array}{c}
P_{1} \\
P_{2} \\
\vdots \\
P_{N}
\end{array}\right], Q_{\sigma}=\left[\begin{array}{llll}
Q_{1} & Q_{2} & \cdots & Q_{N}
\end{array}\right] S_{\sigma}^{*}, \\
P_{i}=\left(\begin{array}{c|c}
A & B \\
\hline C_{i} & D_{i}
\end{array}\right), \text { and } Q_{i}=\left(\begin{array}{c|c}
A & B_{i} \\
\hline C & D_{i}
\end{array}\right) .
\end{gathered}
$$

Notice that for a given switching sequence $\sigma, G_{\sigma} \in \mathscr{S}$ defines a Linear Time Varying (LTV) system. Therefore, $G_{\sigma}$ can be represented by an infinite dimensional lower triangular matrix. By $\mathscr{R}\left[G_{\sigma}\right]_{k}$ and $\mathscr{M}\left[G_{\sigma}\right]_{k}$ we mean the $k$ th row and the $k \times k$ sub-matrix containing elements in the first $k$ rows and columns of the matrix representation $G_{\sigma}$, respectively.

In this article we focus on the model matching problem

$$
\inf _{Q \in \mathscr{S}} \sup _{\left(\sigma_{H}, \sigma_{U}, \sigma_{Q}\right)}\left\|H_{\sigma_{H}}-U_{\sigma_{U}} Q_{\sigma_{Q}}\right\|
$$

where $H_{\sigma_{H}}, U_{\sigma_{U}}$, and $Q_{\sigma_{Q}}$ are switching systems in $\mathscr{S}$.
One application of such a problem can be found in sensitivity minimization. Suppose plant $P_{\sigma_{P}} \in \mathscr{S}$ is a switching plant. Then a large set of stabilizing controllers is given by

$$
K=\left(I-P_{\sigma_{P}} Q_{\sigma_{Q}}\right)^{-1} Q_{\sigma_{Q}},
$$

where $Q_{\sigma_{Q}} \in \mathscr{S}$ is the so-called Youla parameter. Upon substitution of such a parametrization of $K$ in the sensitivity map we obtain

$$
\Phi=I+P_{\sigma_{P}} Q_{\sigma_{Q}}
$$

Hence, the problem of sensitivity minimization under the worst-case switching is reduced to

$$
\inf _{Q \in \mathscr{S}} \sup _{\left(\sigma_{P}, \sigma_{Q}\right)}\left\|I+P_{\sigma_{P}} Q_{\sigma_{Q}}\right\|
$$

In the next sections, we address (2) for different types of switching systems and different assumptions on switching sequences.

\section{Model Matching Problem with INDEPENDENT SWITCHING}

In this section the problem (2) is considered and we assume that the switching sequences $\sigma_{Q}, \sigma_{U}$, and $\sigma_{H}$ can switch independently. Under this assumption, we will show that a switching $Q_{\sigma_{Q}}$ will not provide a better performance over an LTI $Q$ as stated in the following theorem.

Theorem 1: Let $H_{\sigma_{H}}, U_{\sigma_{U}}$, and $Q_{\sigma_{Q}}$ belong to $\mathscr{S}$. If $\sigma_{Q}$ switches independently of $\sigma_{U}$ and $\sigma_{H}$, then

$$
\begin{aligned}
\mu_{0} & :=\inf _{Q \in \mathscr{S}} \sup _{\left(\sigma_{H}, \sigma_{U}, \sigma_{Q}\right)}\left\|H_{\sigma_{H}}-U_{\sigma_{U}} Q_{\sigma_{Q}}\right\| \\
& =\inf _{Z \in \mathscr{L}_{T I}} \sup _{\left(\sigma_{H}, \sigma_{U}\right)}\left\|H_{\sigma_{H}}-U_{\sigma_{U}} Z\right\| .
\end{aligned}
$$

Proof: Let $\varepsilon>0$ be arbitrary. There exists $Q \in \mathscr{S}$ such that

$$
\mu_{0} \leq \sup _{\left(\sigma_{H}, \sigma_{U}, \sigma_{Q}\right)}\left\|H_{\sigma_{H}}-U_{\sigma_{U}} Q_{\sigma_{Q}}\right\|<\mu_{0}+\varepsilon
$$

First we will show that for any $k \in \mathbb{Z}_{+}$,

$$
\begin{aligned}
& \sup _{\left(\sigma_{H}, \sigma_{U}, \sigma_{Q}\right)}\left\|H_{\sigma_{H}}-U_{\sigma_{U}} \Lambda^{-k} Q_{\sigma_{Q}} \Lambda^{k}\right\| \\
& \leq \sup _{\left(\sigma_{H}, \sigma_{U}, \sigma_{Q}\right)}\left\|H_{\sigma_{H}}-U_{\sigma_{U}} Q_{\sigma_{Q}}\right\| .
\end{aligned}
$$


Notice that

$$
\begin{aligned}
& \sup _{\left(\sigma_{H}, \sigma_{U}, \sigma_{Q}\right)}\left\|H_{\sigma_{H}}-U_{\sigma_{U}} Q_{\sigma_{Q}}\right\| \\
& =\sup _{\left(\sigma_{H}, \sigma_{U}, \sigma_{Q}\right) f \neq 0} \sup _{f \neq 0} \frac{\left\|\left(H_{\sigma_{H}}-U_{\sigma_{U}} Q_{\sigma_{Q}}\right) f\right\|}{\|f\|} \\
& \geq \sup _{\left(\sigma_{H}, \sigma_{U}, \sigma_{Q}\right)} \sup _{f \neq 0} \frac{\left\|\left(H_{\sigma_{H}}-U_{\sigma_{U}} Q_{\sigma_{Q}}\right) \Lambda^{k} f\right\|}{\left\|\Lambda^{k} f\right\|} \\
& =\sup _{\left(\sigma_{H}, \sigma_{U}, \sigma_{Q}\right)} \sup _{f \neq 0} \frac{\left\|\Lambda^{-k}\left(H_{\sigma_{H}}-U_{\sigma_{U}} Q_{\sigma_{Q}}\right) \Lambda^{k} f\right\|}{\left\|\Lambda^{k} f\right\|} \\
& =\sup _{\left(\sigma_{H}, \sigma_{U}, \sigma_{Q}\right)}\left\|\Lambda^{-k}\left(H_{\sigma_{H}}-U_{\sigma_{U}} Q_{\sigma_{Q}}\right) \Lambda^{k}\right\| \\
& =\sup _{\left(\sigma_{H}, \sigma_{U}, \sigma_{Q}\right)}\left\|\Lambda^{-k} H_{\sigma_{H}} \Lambda^{k}-\Lambda^{-k} U_{\sigma_{U}} \Lambda^{k} \Lambda^{-k} Q_{\sigma_{Q}} \Lambda^{k}\right\| \\
& =\sup _{\left(\sigma_{H}, \sigma_{U}, \sigma_{Q}\right)}\left\|H_{\Lambda^{-k} \sigma_{H}}-U_{\Lambda^{-k} \sigma_{U}} \Lambda^{-k} Q_{\sigma_{Q}} \Lambda^{k}\right\| \\
& =\sup _{\left(\Lambda^{-k} \sigma_{H}, \Lambda^{-k} \sigma_{U}, \sigma_{Q}\right)}\left\|H_{\Lambda^{-k} \sigma_{H}}-U_{\Lambda^{-k} \sigma_{U}} \Lambda^{-k} Q_{\sigma_{Q}} \Lambda^{k}\right\| \\
& =\sup _{\left(\sigma_{H}, \sigma_{U}, \sigma_{Q}\right)}\left\|H_{\sigma_{H}}-U_{\sigma_{U}} \Lambda^{-k} Q_{\sigma_{Q}} \Lambda^{k}\right\| .
\end{aligned}
$$

Therefore, (5) holds valid. Now define the averaging system

$$
Q_{M}^{\sigma_{Q}}=\frac{1}{M+1}\left\{\sum_{k=0}^{M} \Lambda^{-k} Q_{\sigma_{Q}} \Lambda^{k}\right\} .
$$

Using (5) and the triangle inequality, it is easy to see

$$
\begin{aligned}
& \sup _{\left(\sigma_{H}, \sigma_{U}, \sigma_{Q}\right)}\left\|H_{\sigma_{H}}-U_{\sigma_{U}} Q_{M}^{\sigma_{Q}}\right\| \\
& \leq \sup _{\left(\sigma_{H}, \sigma_{U}, \sigma_{Q}\right)}\left\|H_{\sigma_{H}}-U_{\sigma_{U}} Q_{\sigma_{Q}}\right\| .
\end{aligned}
$$

Then, following the same line of argument as in [6, Theorem 3.1], [7], or [8], there exists a weak* convergent subsequence such that

$$
Q_{L T I}^{\sigma_{Q}}=\lim _{k \rightarrow \infty} w e a k^{*} Q_{M_{k}}^{\sigma_{Q}},
$$

where $Q_{L T I}^{\sigma_{Q}} \in \mathscr{L}_{T I} \subseteq \mathscr{S}$. Moreover,

$$
\sup _{\left(\sigma_{H}, \sigma_{U}\right)}\left\|H_{\sigma_{H}}-U_{\sigma_{U}} Q_{L T I}^{\sigma_{Q}}\right\| \leq \sup _{\left(\sigma_{H}, \sigma_{U}, \sigma_{Q}\right)}\left\|H_{\sigma_{H}}-U_{\sigma_{U}} Q_{\sigma_{Q}}\right\| .
$$

From (4) and (7) in one hand, and the fact that $Q_{L T I}^{\sigma_{Q}} \in \mathscr{S}$ on the other hand, one can write

$$
\mu_{0} \leq \sup _{\left(\sigma_{H}, \sigma_{U}\right)}\left\|H_{\sigma_{H}}-U_{\sigma_{U}} Q_{L T I}^{\sigma_{Q}}\right\| \leq \mu_{0}+\varepsilon,
$$

for every $\varepsilon$ and this completes the proof.

Corollary 2: Consider the sensitivity map

$$
\Phi=I+P_{\sigma_{P}} Q_{\sigma_{Q}}
$$

where $P \in \mathscr{S}_{O}$ is output switching, $Q \in \mathscr{S}$ is any stably switching system, and $\sigma_{Q}$ can switch independent of $\sigma_{P}$. Then

$$
\inf _{Q \in \mathscr{S}} \sup _{\left(\sigma_{P}, \sigma_{Q}\right)}\|\Phi\|=\inf _{Z \in \mathscr{L}_{T I}}\left\|\left[\begin{array}{c}
I \\
\vdots \\
I
\end{array}\right]+\left[\begin{array}{c}
P_{1} \\
\vdots \\
P_{N}
\end{array}\right] Z\right\| .
$$

Proof: The proof is similar to that of [6, Corollary 3.1] and is omitted here.

According to Theorem 1, a switched $Q_{\sigma}$ may result in a better performance only if it has the same switching sequence as the plant. Accordingly, in the next section we consider (2) with $\sigma_{H}=\sigma_{U}=\sigma_{Q}=\sigma$.

\section{Model Matching Problem with Matched SWITCHING}

Although one can hope for a better performance in the case of matched switching sequences, there are cases when such a compensator, with matched switching, still does not result in a better performance over an LTI compensator. It is so, in particular, if the plant is strictly causal as given in the following theorem.

Theorem 3: Let $\sigma$ be a switching sequence, $H_{\sigma} \in$ $\mathscr{S}_{O}$, and $U_{\sigma} \in \mathscr{S}_{O}$ be output switching, and $Q_{\sigma} \in \mathscr{S}$ be any switching system. Further, assume $U_{\sigma}$ is strictly causal. Then

$$
\begin{aligned}
v_{0} & :=\inf _{Q_{\sigma} \in \mathscr{S}} \sup _{\sigma}\left\|H_{\sigma}-U_{\sigma} Q_{\sigma}\right\| \\
& =\inf _{Z \in \mathscr{L}_{T I}} \sup _{\sigma}\left\|H_{\sigma}-U_{\sigma} Z\right\| .
\end{aligned}
$$

Proof: Let $\varepsilon>0$ be arbitrary. Then, there exists $Q_{\sigma} \in \mathscr{S}$ such that

$$
v_{0} \leq \sup _{\sigma}\left\|H_{\sigma}-U_{\sigma} Q_{\sigma}\right\|<v_{0}+\varepsilon .
$$

Now we will show that

$$
\begin{aligned}
v_{k} & :=\sup _{\sigma}\left\|H_{\sigma}-U_{\sigma} \Lambda^{-k} Q_{\sigma} \Lambda^{k}\right\| \\
& \leq \sup _{\sigma}\left\|H_{\sigma}-U_{\sigma} Q_{\sigma}\right\|, \text { for } \forall k \in \mathbb{Z}_{+} .
\end{aligned}
$$

Once (8) is established, the rest of the proof follows analogous to the proof of Theorem 1 from equation (6) on. To show (8), since the associated norm is the $l_{\infty}$ induced norm, for any $\varepsilon^{\prime}>0$, there exist a switching sequence $\sigma^{\prime}$ and $t^{\prime} \geq 0$ such that

$$
v_{k}-\varepsilon^{\prime}<\left|\mathscr{R}\left[H_{\sigma^{\prime}}-U_{\sigma^{\prime}} \Lambda^{-k} Q_{\sigma^{\prime}} \Lambda^{k}\right]_{t^{\prime}}\right|_{l_{1}} \leq v_{k} .
$$

Defined a sequence $\bar{\sigma}($.$) as$

$$
\bar{\sigma}(t)=\left\{\begin{array}{cc}
\sigma^{\prime}(t+k) & \text { for } t \neq t^{\prime} \\
\sigma^{\prime}\left(t^{\prime}\right) & \text { for } t=t^{\prime}
\end{array}\right.
$$


Then, one can write

$$
\begin{aligned}
& \mathscr{R}\left[H_{\sigma^{\prime}}-U_{\sigma^{\prime}} \Lambda^{-k} Q_{\sigma^{\prime}} \Lambda^{k}\right]_{t^{\prime}} \\
= & \mathscr{R}\left[H_{\sigma^{\prime}}\right]_{t^{\prime}}-\mathscr{R}\left[U_{\sigma^{\prime}}\right]_{t^{\prime}} \mathscr{M}\left[\Lambda^{-k} Q_{\sigma^{\prime}} \Lambda^{k}\right]_{t^{\prime}} \\
= & \mathscr{R}\left[H_{\sigma^{\prime}}\right]_{t^{\prime}}-\mathscr{R}\left[U_{\sigma^{\prime}}\right]_{t^{\prime}}\left[\begin{array}{c}
\mathscr{M}\left[\Lambda^{-k} Q_{\sigma^{\prime}} \Lambda^{k}\right]_{t^{\prime}-1} \mathbf{0} \\
\mathscr{R}\left[\Lambda^{-k} Q_{\sigma^{\prime}} \Lambda^{k}\right]_{t^{\prime}}
\end{array}\right] \\
= & \mathscr{R}\left[H_{\bar{\sigma}}\right]_{t^{\prime}}-\mathscr{R}\left[U_{\bar{\sigma}}\right]_{t^{\prime}}\left[\begin{array}{c}
\mathscr{M}\left[\Lambda^{-k} Q_{\sigma^{\prime}} \Lambda^{k}\right]_{t^{\prime}-1} \mathbf{0} \\
\mathscr{R}\left[\Lambda^{-k} Q_{\sigma^{\prime}} \Lambda^{k}\right]_{t^{\prime}}
\end{array}\right],
\end{aligned}
$$

where $\mathbf{0}$ is a zero matrix with the same number of columns as $\mathscr{M}\left[\Lambda^{-k} Q_{\sigma^{\prime}} \Lambda^{k}\right]_{t^{\prime}-1}$. Notice that, $\quad \mathscr{M}\left[\Lambda^{-k} Q_{\sigma^{\prime}} \Lambda^{k}\right]_{t^{\prime}-1}=\mathscr{M}\left[Q_{\bar{\sigma}}\right]_{t^{\prime}-1}$, but $\mathscr{R}\left[\Lambda^{-k} Q_{\sigma^{\prime}} \Lambda^{k}\right]_{t^{\prime}} \neq \mathscr{R}\left[Q_{\bar{\sigma}}\right]_{t^{\prime}}$ if $\sigma^{\prime}\left(t^{\prime}+k\right) \neq \sigma^{\prime}\left(t^{\prime}\right)$. Also, notice that since $U_{\bar{\sigma}}$ is strictly causal the outcome of

$$
\mathscr{R}\left[U_{\bar{\sigma}}\right]_{t^{\prime}}\left[\begin{array}{c}
\mathscr{M}\left[\Lambda^{-k} Q_{\sigma^{\prime}} \Lambda^{k}\right]_{t^{\prime}-1} \mathbf{0} \\
\mathscr{R}\left[\Lambda^{-k} Q_{\sigma^{\prime}} \Lambda^{k}\right]_{t^{\prime}}
\end{array}\right]
$$

does not depend on $\mathscr{R}\left[\Lambda^{-k} Q_{\sigma^{\prime}} \Lambda^{k}\right]_{t^{\prime}}$ and hence one can write

$$
\left|\mathscr{R}\left[H_{\sigma^{\prime}}-U_{\sigma^{\prime}} \Lambda^{-k} Q_{\sigma^{\prime}} \Lambda^{k}\right]_{t^{\prime}}\right|_{1}=\left|\mathscr{R}\left[H_{\bar{\sigma}}-U_{\bar{\sigma}} Q_{\bar{\sigma}}\right]_{t^{\prime}}\right|_{1} .
$$

Moreover

$$
\begin{aligned}
\left|\mathscr{R}\left[H_{\bar{\sigma}}-U_{\bar{\sigma}} Q_{\bar{\sigma}}\right]_{t^{\prime}}\right|_{1} \leq\left\|H_{\bar{\sigma}}-U_{\bar{\sigma}} Q_{\bar{\sigma}}\right\|_{l_{\infty \infty}-i n d} \\
\leq \sup _{\sigma}\left\|H_{\sigma}-U_{\sigma} Q_{\sigma}\right\|_{l_{\infty}-i n d} .
\end{aligned}
$$

Therefore,

$$
v_{k}-\varepsilon^{\prime} \leq \sup _{\sigma}\left\|H_{\sigma}-U_{\sigma} Q_{\sigma}\right\|,
$$

for any $\varepsilon^{\prime}>0$ and this proves (8).

Theorem 3 does not hold valid if $U_{\sigma}$ is not strictly causal. As a matter fact, in [6], it is shown that a better performance may be achieved in the case of matched switching if $U_{\sigma}$ is not strictly causal where the authors studied the problem (2) with $H_{\sigma}, U_{\sigma}$, and $Q_{\sigma}$ being output switching. We extend their results, in what follows, and consider (2) with $H_{\sigma}$ and $U_{\sigma}$ being output switching and $Q_{\sigma}$ being input switching with matched switching sequences.

For simplicity of notations, assume $H_{\sigma}, U_{\sigma}$, and $Q_{\sigma}$ switch between two modes. Then, $H_{\sigma}-U_{\sigma} Q_{\sigma}$ can be written as

$$
\begin{aligned}
& S_{\sigma}\left[\begin{array}{l}
H_{1} \\
H_{2}
\end{array}\right]-S_{\sigma}\left[\begin{array}{c}
U_{1} \\
U_{2}
\end{array}\right]\left[\begin{array}{ll}
Q_{1} & Q_{2}
\end{array}\right] S_{\sigma}^{*} \\
= & S_{\sigma}\left[\begin{array}{cc}
H_{1} & H_{1} \\
H_{2} & H_{2}
\end{array}\right] S_{\sigma}^{*}-S_{\sigma}\left[\begin{array}{c}
U_{1} \\
U_{2}
\end{array}\right]\left[\begin{array}{ll}
Q_{1} & Q_{2}
\end{array}\right] S_{\sigma}^{*} \\
= & S_{\sigma}\left[\begin{array}{cc}
H_{1}-U_{1} Q_{1} & H_{1}-U_{1} Q_{2} \\
H_{2}-U_{2} Q_{1} & H_{2}-U_{2} Q_{2}
\end{array}\right] S_{\sigma}^{*}
\end{aligned}
$$

and thus (2) is equivalent to

$$
\inf _{\substack{Q_{1} \in \mathscr{L}_{T I} \\
Q_{2} \in \mathscr{L}_{T I}}} \sup _{\sigma}\left\|S_{\sigma}\left[\begin{array}{cc}
H_{1}-U_{1} Q_{1} & H_{1}-U_{1} Q_{2} \\
H_{2}-U_{2} Q_{1} & H_{2}-U_{2} Q_{2}
\end{array}\right] S_{\sigma}^{*}\right\| \text {. }
$$

Notice that (9) is an input-output switching system. In [1], the worst-case $l_{\infty}$-induced norm of a SISO inputoutput switching system is computed. In the next section, the results of [1] are generalized to MIMO inputoutput systems and expressions to compute the worstcase $l_{\infty}$-induced norm is presented. Once those formulas are in hand, one can see that the problem of minimizing (10) is convex and in fact a linear programming.

\section{WORST-CASE $l_{\infty}$-INDUCED NORM COMPUTATION}

In this section, we present our results regarding the computation of $l_{\infty}$ induced norm for input-output switching systems. Consider the input output switching system

$$
G_{\sigma}:\left\{\begin{array}{l}
x(t+1)=A x(t)+B_{\sigma(t)} u(t) \\
y(t)=C_{\sigma(t)} x(t)+D_{\sigma(t)} u(t)
\end{array} .\right.
$$

We are interested in finding the worst-case $l_{\infty}$ induced norm defined as

$$
\|G\|:=\sup _{\sigma}\left\|G_{\sigma}\right\|
$$

It is easy to see that the problem of finding the worstcase $l_{\infty}$ induced norm of a system with $m$ inputs and $p$ outputs can be reduced to $p$ independent problems of systems with $m$ inputs and a single output.

Define the LTI system $H_{i j}^{r}$ to have the realization $H_{i j}^{r}=\left(\begin{array}{c|c}A & B_{j} \\ \hline C_{i}(r) & D_{i}(r)\end{array}\right)$ with impulse response of

$$
\left(H_{i j}^{r}(t)\right)_{t=0}^{\infty}=\left\{\begin{array}{cc}
D_{i}(r) & \text { for } t=0 \\
C_{i}(r) A^{t-1} B_{j} & \text { for } t>0
\end{array},\right.
$$

where $C_{i}(r)$ and $D_{i}(r)$ is the $r$ th row of $C_{i}$ and $D_{i}$ respectively.

Theorem 4: For the multi input multi output system (11), the worst-case $l_{\infty}$ induced norm can be calculated as

$$
\|G\|=\max _{r \in\{1, \ldots, p\}} \max _{j \in \mathbb{Z}_{N}}\left|\bar{g}_{j}^{r}\right|_{1}
$$

where the sequence $\left(\bar{g}_{j}^{r}(n)\right)_{n=0}^{\infty}$ is defined as

$$
\bar{g}_{j}^{r}(n)=\left\{\begin{array}{cl}
\left|H_{j j}^{r}(0)\right|_{1} & \text { for } n=0 \\
\max _{k \in \mathbb{Z}_{N}}\left|H_{j k}^{r}(n)\right|_{1} & \text { for } n>1
\end{array}\right. \text {. }
$$

Proof: For a given switching sequence $\sigma$, let $G_{\sigma}^{r}$ be the operator from input $u$ to the $r$ th output. That is,

$$
y_{r}=G_{\sigma}^{r} u
$$

where $y_{r}$ is the $r$ th entry of

$$
y=\left(y_{1}, y_{2}, \ldots, y_{p}\right)^{T} .
$$


One can visualize the operator $G_{\sigma}^{r}$ as a lower triangular infinite dimensional matrix

$$
\begin{aligned}
& G_{\sigma}^{r}= \\
& {\left[\begin{array}{ccccc}
H_{\sigma(0) \sigma(0)}^{r}(0) & & & & \\
H_{\sigma(1) \sigma(0)}^{r}(1) & H_{\sigma(1) \sigma(1)}^{r}(0) & & \\
H_{\sigma(2) \sigma(0)}^{r}(2) & H_{\sigma(2) \sigma(1)}^{r}(1) & H_{\sigma(2) \sigma(2)}^{0}(0) & \\
\vdots & \vdots & \vdots & \ddots
\end{array}\right]}
\end{aligned}
$$

The worst-case $l_{\infty}$ induced norm for this operator is given by

$$
\begin{aligned}
& \left\|G^{r}\right\|=\sup _{t} \sup _{\sigma} \\
& \left|\left[H_{\sigma(t) \sigma(0)}^{r}(t) H_{\sigma(t) \sigma(1)}^{r}(t-1) \ldots H_{\sigma(t) \sigma(t)}^{r}(0)\right]\right|_{1} .
\end{aligned}
$$

Therefore, for any $\varepsilon>0$, there exists $t \in \mathbb{Z}_{+}$such that

$$
\begin{aligned}
& \left\|G^{r}\right\| \geq \sup _{\sigma} \\
& \left|\left[\begin{array}{llll}
H_{\sigma(t) \sigma(0)}^{r}(t) & H_{\sigma(t) \sigma(1)}^{r}(t-1) & \ldots H_{\sigma(t) \sigma(t)}^{r}(0)
\end{array}\right]\right|_{1} \\
& >\|G\|-\varepsilon \text {. }
\end{aligned}
$$

Notice that the value of $\sigma(t) \in \mathbb{Z}_{N}$ has finite number of possibilities and does not affect the value of $\sigma(k)$ for $k<t$. Thus, we can write

$$
\begin{aligned}
& \left\|G^{r}\right\| \geq \max _{j \in \mathbb{Z}_{N} \sigma} \sup _{\sigma} \\
& \left|\left[H_{j \sigma(0)}^{r}(t) H_{j \sigma(1)}^{r}(t-1) \ldots H_{j j}^{r}(0)\right]\right|_{1} \\
& >\left\|G^{r}\right\|-\varepsilon .
\end{aligned}
$$

Since $\left|H_{j \sigma(0)}^{r}(t) H_{j \sigma(1)}^{r}(t-1) \ldots H_{j j}^{r}(0)\right|_{1}$ is a row matrix, one can write

$$
\begin{aligned}
& \max _{j \in \mathbb{Z}_{N} \sup _{\sigma}} \\
& \left|\left[H_{j \sigma(0)}^{r}(t) H_{j \sigma(1)}^{r}(t-1) \ldots H_{j j}^{r}(0)\right]\right|_{1} \\
= & \max _{j \in \mathbb{Z}_{N}} \sup _{\sigma}\left(\sum_{\tau=0}^{t}\left|H_{j \sigma(\tau)}^{r}(t-\tau)\right|_{1}\right) \\
= & \max _{j \in \mathbb{Z}_{N}}\left(\sum_{\tau=0}^{t} \sup _{\sigma(\tau) \in \mathbb{Z}_{N}}\left|H_{j \sigma(\tau)}^{r}(t-\tau)\right|_{1}\right) \\
\leq & \max _{j \in \mathbb{Z}_{N}}\left|\bar{g}_{j}^{r}\right|_{1} \cdot
\end{aligned}
$$

Therefore,

$$
\max _{j \in \mathbb{Z}_{N}}\left|\bar{g}_{j}^{r}\right|_{1}>\left\|G^{r}\right\|-\varepsilon, \text { for any } \varepsilon>0
$$

and obviously

$$
\max _{j \in \mathbb{Z}_{N}}\left|\bar{g}_{j}^{r}\right|_{1} \leq\left\|G^{r}\right\|
$$

which implies

$$
\left\|G^{r}\right\|=\max _{j \in \mathbb{Z}_{N}}\left|\bar{g}_{j}^{r}\right|_{1}
$$

The proof is complete by noticing that

$$
\|G\|=\max _{r \in\{1,2, \ldots, p\}}\left\|G^{r}\right\|=\max _{r \in\{1,2, \ldots, p\}} \max _{j \in \mathbb{Z}_{N}}\left|\bar{g}_{j}^{r}\right|_{1} .
$$

In the light of Theorem 4, one can cast the problem (10) as a linear programming which can be solved effectively using the available commercial software.

\section{CONCLUSION}

We considered model matching problems with the systems involved being linear switched systems. It was shown that switching compensators cannot over-perform LTI compensators in the case of unmatched switching sequences or even in the case of matched switching when the plant is strictly causal. In the case of matched switching with $H$ and $U$ being output switching and causal and $Q$ being input switching, the problem of finding the optimal $Q$ can be cast as linear programming using the exact expression derived for the $l_{\infty}$ gain of the input-output switching system.

\section{REFERENCES}

[1] P. Voulgaris, "On the computation of $l_{\infty}$ gains of switched systems," in 50th IEEE Conference on Decision and Control and European Control Conference (CDC-ECC) Orlando, FL, USA, Dec 2011.

[2] A. Rantzer and M. Johansson, "Piecewise linear quadratic optimal control," Automatic Control, IEEE Transactions on, vol. 45, no. 4, pp. 629-637, 2000.

[3] D. Liberzon, Switching in systems and control. Springer, 2003.

[4] J. Lee and P. Khargonekar, "Optimal output regulation for discrete-time switched and markovian jump linear systems," SIAM Journal on Control and Optimization, vol. 47, no. 1, pp. 40-72, 2008.

[5] H. Lin and P. Antsaklis, "Stability and stabilizability of switched linear systems: a survey of recent results," Automatic Control, IEEE Transactions on, vol. 54, no. 2, pp. 308-322, 2009.

[6] S. Jiang and P. Voulgaris, "Performance optimization of switched systems: a model matching approach," Automatic Control, IEEE Transactions on, vol. 54, no. 9, pp. 2058-2071, 2009.

[7] H. Chapellat and M. Dahleh, "Analysis of time-varying control strategies for optimal disturbance rejection and robustness," Automatic Control, IEEE Transactions on, vol. 37, no. 11, pp. 17341745, 1992.

[8] J. Shamma and M. Dahleh, "Time-varying versus time-invariant compensation for rejection of persistent bounded disturbances and robust stabilization," Automatic Control, IEEE Transactions on, vol. 36, no. 7, pp. 838-847, 1991. 